\title{
FAMILIAL ECTOPIA LENTIS AND ITS COMPLICATIONS*
}

\author{
BY \\ E. T. MEYER \\ Johannesburg, Union of S. Africa
}

Familial ectopia lentis is a well recognized condition and is usually seen by the ophthalmologist in its fully developed stage. It was thought worth while to record this family, as some of the very earliest stages have been observed and the complications of the condition have been very varied. The family is a large one, spread over a large part of the Union of South Africa, and it has not been possible to see as many of its members as one would wish. Much of the evidence presented in the family tree was given by one of the older members of the family, herself a sufferer from the condition, who seemed to have made it her business to find out about the vision of her relatives. Some of the patients described below consulted my father over a period of many years, and it is only more recently that I have had the opportunity of seeing them myself and of watching the onset and the course of the condition in one of the younger members. Several of the patients only sought advice in the later stages of the condition, by which time there was little or no sight to be retrieved.

\section{Features of the Condition}

Most of the complications of this condition which have been described are presented by one or other member of this family, so that a resumé of the main features of the condition will include those found in these cases. Marfan's syndrome, first described in 1896 without ocular changes, comprises the physical features of elongated long bones (especially those of the hands and feet) and spinal curvatures; it is of interest that the members of this family are all tall, many being over $6 \mathrm{ft}$. in height, but that arachnodactyly is not present.

A good many of the patients in this family had auburn or red hair, and were of the opinion that this colouring was associated with the ocular abnormalities. I have not been able to substantiate this supposition; as far as I can tell, the colour of hair gives no easy guide to their ocular troubles.

Early Descriptions. - Congenital subluxation of the lens has a strong hereditary background, being usually transmitted as a dominant characteristic. It was described by von Graefe (1854), and Morton (1879) gave examples transmitted through five generations. The literature suggests that it is almost four times more frequent amongst males than amongst-females, but this finding is not confirmed by the

*Received for publication November 2, 1953. 
present series. The condition occurs in this family during the fourth and fifth decades of life, with a tendency to anticipation in the younger generations.

Cause.-This appears to be a degeneration of the zonular fibres. Once the zonule has weakened, dislocation becomes easier by the mere weight of the lens or by minor traumata. The zonular fibres degenerate at the edge of the lens, so that the free margin of the lens can often be seen. Associated with the zonular degeneration, the vitreous also tends to liquefy, and herniation of vitreous may occur through the zonular defect into the anterior chamber.

Progress.-This is well demonstrated by the case of III, 10, who was an Air Force pilot and as such presumably had excellent unaided vision in each eye. He was also a first-class rifle shot, who regularly took part in competitive range shooting, and when first seen his only complaint was difficulty in seeing targets on the shooting range. He had been examined for flying two months earlier, and had been told then that the vision in one eye had deteriorated to $6 / 9$ from its previous $6 / 6$. When seen by me his vision had deteriorated further to $6 / 36$ and $6 / 24$, but with a correction for myopia and astigmatism it was restored to 6/6. At this time he showed iridodonesis of both irides, not throughout their whole extent but localized to two-thirds of the circumference of the limbus. During the next few months the iridodonesis became total. The relaxation of the suspensory mechanism of the lens resulted in a lenticular myopia as the lens assumed a more spherical shape. His astigmatism became more marked, and one assumes that this was due to tilting of the lens and to unequal traction of the zonule in its different parts as the zonular fibres were degenerating. The lens is described as being most commonly displaced sideways in these conditions, but this displacement is as yet small in his case.

The usual deep anterior chamber was well demonstrated in his case at this stage. Such subluxated lenses may then produce effects other than those due to the changes of refraction. The lens may leave part of the pupil aphakic, and produce a most annoying set of images, as in one case in this series. The subluxated lenses may become opaque, and, although this is stated to be relatively rare, cataract has been a common occurrence in the family. More usually a secondary glaucoma follows, or an irritative iridocyclitis is set up. The reason for the occurrence of such a glaucoma has been a matter for speculation as such cases are usually seen with deep anterior chambers. In the case of III, 10, the previously deep anterior chambers have become extremely shallow, and iridodonesis is no longer easily evident. One feels that he must now be approaching a stage at which glaucoma will become manifest; the refraction has become progressively more myopic the ocular tension is normal, the fields are full, and the discs are not cupped.

For further changes one must look at other members of the family. Complete dislocation of the lens may occur, and when this takes place into the anterior chamber it may produce glaucoma, either when the lens is impacted in the pupil or when it enters completely into the anterior chamber (Heath, 1941). This complication is best treated by mydriatics and posture in an attempt to get the lens back through the pupil, and if necessary by subsequent extraction. Such a case is IV, 12, whose second eye seems perfectly normal so far. Posterior dislocation, not seen in this family, has been described in the literature, and the cataractous lens may lie in the vitreous sometimes for years without any other ill-effects.

Ocular Tension.-The literature suggests that a rise in tension is not very commonly associated with subluxation of the lens, and if one accepts this view, then the 
relatively large number of cases of glaucoma in this family may be due to a more total dislocation of the lens than is usually present, the resultant acute glaucoma being responsible for the poor visual results. The best treatment for such cases, if the lens does not return through the pupil after using mydriatics, is intracapsular lens extraction after the tension has been reduced to as normal a level as possible.

\section{Material}

The first evidence of the condition in this family was found in I, 2, mother of II, 2,3 , and 4 , the three oldest cases examined in the series; she was not seen by us, but her eldest daughter (II, 2) reported that she had always been short-sighted and had been told in 1917 that she had dislocated lenses and glaucoma. She had emigrated from Europe to $S$. Africa many years ago, so that it is unlikely that the affliction can be traced further back.

I, 2 had altogether two sons and four daughters, all with poor sight. The elder son (II, 1) had cataracts, and one of his three sons had poor sight, though the reason for this is not available. The next three children (II, 2, 3, and 4) had dislocated lenses, cataracts, and glaucoma. The two youngest daughters (II, 5 and 6), both of whom died in their forties, had poor eyesight for which operations were undertaken.

The youngest of the four daughters (II, 6) had two children whose eyesight, as far as is known, is satisfactory.

The next youngest daughter (II, 5) had five children (III,

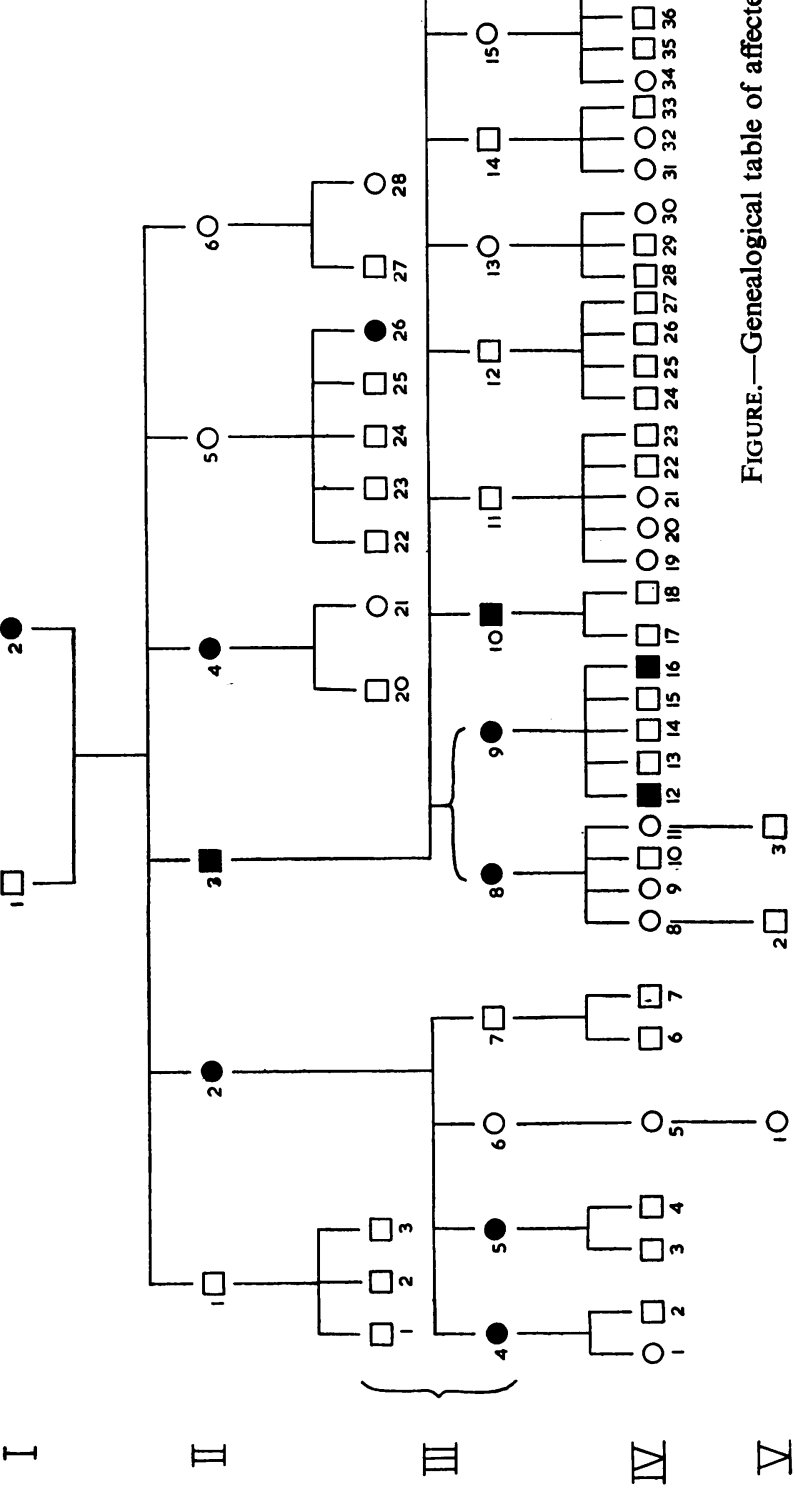
22-26) only one of whom (III, 
26) appears to have had affected eyes; she was highly myopic with lenses dislocated up and in, and at the age of 31 years had a retinal detachment.

The third youngest daughter (II, 4) was known to have had glaucoma, dislocated lenses, and cataracts, and to have had successful operations performed on her eyes. She had two children (III, 20 and 21) both with " poor eyes".

We now come to the two branches of the family about which we know most; that of II, 2 and that of II, 3. Of those previously referred to, only III, 26 was seen by us, the others having been described from information given by relatives.

(1) II, 2, female born in 1878, had her right eye enucleated in 1932 for an absolute glaucoma brought about by a cataractous dislocated lens. Shortly afterwards, she developed acute glaucoma in the left eye and this failed to respond to the miotics used. At this stage she was referred to my father, who reduced the tension by a trephine operation with a complete iridectomy, after which her vision was $6 / 9$ with a correction. She was not seen by him thereafter for 10 years, until in 1942 she was referred back to him, and he extracted the lens intracapsularly; the corrected vision was then $6 / 6$, the field full, and the disc normal. In the 10 years during which she has since been followed up, the disc has remained normal, and vision a year ago was $6 / 9$ with some commencing senile macular changes.

This patient has three daughters and one son (III, 4-7); of these, two daughters (III, 4 and III, 5) show dislocated lenses, and the other daughter and son (III, 6 and III, 7) are unaffected so far as we know.

The eldest daughter (III, 4) has always been short-sighted, and since the age of 44 has shown some lens striae. Her myopia has increased from -1.5 D.sph. -0.75 D.cyl., and -2 D.sph. -0.5 D. cyl., when first seen in 1946, to - 4 D.sph.-2.75 D. cyl., and - 3 D.sph.-2 D. cyl., when last seen in January, 1953. Her accommodative power has been poor; she required a +1.75 D.sph. for near sight in 1946, and to-day she requires an added +3 D.sph. to enable her to read J 1. There is as yet no iridodonesis, but at her most recent visit the internal edges of the lenses could be seen, indicating that the lenses had dislocated slightly temporally. The interest in her case lies in the fact that there have been lens striae for some years now, in the ever increasing myopia, especially the astigmatic error, and extremely poor accommodation. In retrospect, knowing the family, these features indicate a laxity of the zonule, and the edges of the lenses can now be seen, indicating that the lenses are displaced.

The other affected daughter (III, 5), born in 1911, was told at the age of 7 years that she had " undeveloped lenses". In 1923 at the age of 13 she had already had the left lens needled on three occasions, and the right lens was dislocated upwards and inwards. She was not seen again by my father until 1943 when he extracted the right cataract, and the remnants of the left lens. Since then her vision has been correctable to $6 / 9$ in each eye. In November, 1952, the maculae showed degenerative changes, but discs were still quite normal.

The seven grandchildren (IV, 1-7), ranging up to the age of 30 years, and one great-grandchild $(\mathrm{V}, 1)$, aged 9 , all have satisfactory sight.

(2) II, 3, male, born in 1880, was told in 1914 that he had dislocated lenses. The same diagnosis was made in 1921, and in 1925 and 1926 he had operations to his eyes for the cataracts that had by then developed. He seems to have had a series of post-operative mishaps and his history gives some idea of the difficulties his surgeons encountered. When first seen by my father and myself at the end of 1951 , he was aphakic, the visual acuity being 6/36 with correction in the right eye, and counting fingers at $1 \mathrm{~m}$. in the left eye: both discs were pale and deeply cupped.

This patient has a family of six sons and six daughters (III 8-19), of whom two twin daughters (III, 8 and 9) born in 1903, and one son (III, 10) have been seen by us. Of the others, one daughter (III, ?) has " one very poor eye ", but, as she has not been seen, the cause is not known; two sons (III, ? and ?) have " impaired vision", the reason being also not known to us.

The two sisters III, 8 and 9 appear to be uniovular twins. III, 8 apparently had no ocular trouble until in April, 1950, when she noticed that her vision was becoming blurred, and glasses were prescribed but soon seemed unsatisfactory. Later in the year she developed pain in the right eye, and in November, 1950, this eye was trephined. The same night the left eye became painful 
and a month later a complete iridectomy was performed on the left eye. She was first seen by me in 1951, by which time vision was reduced to perception of light, bilateral dislocated lenses with cataracts were present, and the tension was raised. Cyclodialyses were done to improve the tension and the cataracts were extracted. The visual result was not a great improvement but the eyes have remained quiet and the tension satisfactory.

Of her four children (IV 8-11) one has weak eyes requiring glasses, but I have no further definite information about them.

III 9, the second twin, first had an attack of glaucoma with loss of sight of the right eye in 1948. The left eye gave no trouble until July, 1951, an attack of glaucoma developed, and she had an operation (?trephine). She was first seen by me in September, 1951, when neither eye had an anterior chamber and both lenses were cataractous and dislocated. A cyclodialysis, and later extraction, was done on the left eye, and vision improved slightly from perception of light to hand movements at $2 \mathrm{ft}$. A corneal dystrophy, one of the complications of this type of condition, has subsequently also become worse.

Of her five sons (IV, 12-16), three have no ocular complaints; one of the three aged 28, has been examined, and shows no signs of dislocated lenses.

The youngest son (IV, 16), born in 1927, has had a "lazy" left eye as long as he can remember and has worn glasses since he was 10 years old. Both anterior chambers are deep.

The right iris shows an area of iridodonesis in the lower nasal quadrant, and vision is correctable to $6 / 5$ with -0.75 D.sph. -1.5 D. cyl. at $90^{\circ}$.

The left eye is $10^{\circ}$ divergent, and the iris shows iridodonesis over its whole surface. The lower edge of the lens is visible at times in the lower part of the pupil, the field is full and the discs normal, and vision can be corrected to $6 / 18$ with -12 D.sph. -3 D.cyl. at $45^{\circ}$.

The eldest son (IV, 12), born in 1922, had good sight until the end of 1948 when the vision of the left eye became blurred.

In October, 1951, he was told that he had a dislocated lens, and vision in the left eye was reduced to 5/60, whereas on his discharge from the Army in 1945 he had been able to read 6/6.

The right eye appeared to be normal but required -2 D.cyl. to read 6/6. The left eye showed a shallow anterior chamber, with the lens dislocated downwards and inwards, and vision correctable to 6/9 with-7 D.sph.-1 D. cyl. At times he had pain of a glaucomatous nature in the left eye and this had not improved with the use of miotics. The refraction had changed further so that in May, 1953, vision in the right eye was 6/9 with -3.5 D. cyl., and in the left 6/12 with - 14 D.sph. -3 D.cyl.

Of the 37 grandchildren (IV 8-44) two (V, 12 and 16) are so far known to have had dislocated lenses, and a third has suspect eyes. A full investigation of all these grandchildren might well produce many more cases.

I think the case of III, 10, born in 1916, the third child of II, 3 to be afflicted with the condition, is the most instructive, as the earliest stages of the condition can be traced. He was a distinguished pilot in the Air Force, with a sound career both non-operational and operational behind him, and a first-class rifle shot (having taken many prizes at the ranges), and these features bear out his claim that when he was examined routinely for flying in 1950 the vision in each eye was 6/6. In 1951 he was told that the vision of the right eye had deteriorated a little to $6 / 9$ but nobody took much notice of this at the time. In September, 1951, he began to notice difficulty in seeing the target at the shooting range; this first brought him in touch with ophthalmologists, and his sight became so poor that he was eventually discharged from the forces.

In December, 1951, he had deep anterior chambers with iridodonesis of two-thirds of both irides. There was a suggestion of transverse folds in the posterior capsule of the left lens. Unaided vision was $6 / 36$ in the right eye and $6 / 24$ in the left, and could be corrected to $6 / 5$ with -1.5 D.sph. $-0.5 \mathrm{D}$. cyl. at $180^{\circ}$ for the right eye, and -1.25 D.sph. -1 D.cyl. at $180^{\circ}$ for the left eye. A month later he required an extra - 1 D.sph. for each eye to restore his vision to $6 / 5$, and in another month his correction to attain $6 / 6$ was -3 D.sph. -1.5 D.cyl. at $85^{\circ}$ for the right eye, and -4.5 D. sph. $-0.5 \mathrm{D}$. cyl. at $20^{\circ}$ for the left eye. Six months later his correction had further altered to -6 D. sph. $-1.75 \mathrm{D}$. cyl. at $70^{\circ}$ right and $-6 \mathrm{D}$. sph. $-1.5 \mathrm{D}$. cyl. at $85^{\circ}$ left. Thus during the 8 months in which he was under observation, his myopia had increased by more than four dioptres, and the axis of his cylinder had changed from horizontal to more or less vertical. During this time he also required a reading correction and used one of his weaker pairs of glasses for this purpose. 
When he was seen next in the middle of November, 1952, a change in the physical findings had occurred. His refraction had not changed much in the right eye, but the left eye now required -6.5 D.sph. -2 D. cyl. at $95^{\circ}$ to attain $6 / 9$ vision. The striking feature now was that whereas the anterior chambers had previously been deep, they were now very shallow, and his pupils were larger than one had become accustomed to in his case. There was now no iridodonesis present, and the discs and tension were normal. In March, 1953, there was but little change, and his blind spots were of a normal size.

The problem now to be faced in his case is whether he has reached the stage at which glaucoma attacks are to be expected, and if so what is the best line of treatment to be adopted. Had he been seen for the first time in November, 1952, it would have been extremely difficult to diagnose the presence of subluxated lenses clinically. His twin sisters (II, 8-9) reached the glaucoma stage before they sought advice and the results of operative treatment were extremely disappointing. His aunt (II, 2), was successfully relieved of symptoms by a trephine and only had the lens extracted about 10 years later.

\section{Discussion}

Relationship with Marfan's Syndrome.-In the literature, one finds extensive accounts of ectopia lentis associated with arachnodactyly and ether lesions and abnormalities usually referred to as Marfan's syndrome. This syndrome was exhaustively reviewed by Rados (1942), who summarized the cases reported up to that time, and later by Ross (1949), who collected a further 117 cases. Between them these authors collected the findings in 321 cases. The present series does not fit into the classification of Marfan's syndrome, nor into the type known as Marchesani's syndrome, about which little appears in the literature, where the distinguishing features are the presence of brachycephalic skulls (instead of the usual dolichocephalic skulls found in cases of Marfan's syndrome), short fat limbs, and spherophakia.

The findings can be grouped into defects of three systems:

(1) Skeletal Defects.-These comprise dolichocephalic skulls with prominent supraorbital ridges, frontal bossing, and broad sunken noses. The palatal arch is narrow and the chin prominent and massive. The face is thin with a wrinkled skin and the patients look prematurely aged and have a melancholy or pained expression. The ears are often malformed. The limbs are long and slender, the span between the fingertips of the outstretched arms being greater than the height of the individual. The bones of the hands and feet are elongated and there may be webbing of the fingers. The chest may be funnel shaped or flattened, and kyphosis and scoliosis are common. The scapulae are often winged, and the joints, especially the elbows, fingers, and knees, are lax and subluxation may occur. About 75 per cent. of the cases in Rados's and Ross's series had skeletal changes, 40 per cent. high arched palates, and 20 per cent. abnormal ears.

(2) General and Visceral Defects. - The muscles are poorly developed and there is an absence of subcutaneous fat which makes these patients look taller than they really are. The cardiovascular lesions seem to be the most serious in the lives of the patients, but not much stress is laid on these in the ophthalmological text-books. The incidence in Marfan's syndrome is about 20 per cent., but there tends to be a paucity of symptoms referable to cardiovascular lesions. In the heart, patent foramen ovale, and inter-auricular and interventricular defects are the commonest lesions; fenestration of the aortic valves, coarctation of the aorta, cardiac failure, and patent ductus arteriosus also occur (Lambie, Shellshear, and Shellshear, 1950). The aorta often shows hypoplasia with aneurysm formation and aortic incompetence, and fatal rupture of the aorta has occurred. Of a group of 23 cases of arachnodactyly that have come to autopsy, thirteen had valvular lesions, eleven aortic disease with aneurysm formation (and rupture of the aneurysm in 4 cases), and eight had congenital lesions of the inter-atrial septum (Reynolds, 1950; Fishl and Ruthberg, 1951). The cardiovascular lesions are caused by a deficiency in the elastic tissue 
of the media of the blood vessels, but whether this is due to a mesoblastic developmental defect, or only arises later in life as the result of intrinsic defects in the vasa vasorum of the larger vessels, is not known. The aorta may show cystic and mucinous degeneration of the media, and a resulting aneurysm represents the most serious complication of this disease, often proving fatal in early adult life. The degenerative disposition may not be confined to the aorta, but may extend to the larger arteries of the extremities (Lindeboom and Westerveld-Brandon, 1950). Marvel and Genovese (1951) report aortic aneurysms found in fifteen of 28 cases at autopsy, eight being dissecting aneurysms.

In addition, these patients seem to have a cardiovascular system especially prone to the ravages of rheumatic fever.

Besides the cardiovascular lesions, abnormal configurations of the lungs have been found, with variations in the segmental lobular development. Vestigial lobes are found in the lungs; the right lung may show only two lobes, or the left lung only one (Hamwi, 1951); and these cases are said to be predisposed to respiratory infections. It is, then, these cardiovascular and respiratory lesions which are the danger to life in cases of Marfan's syndrome.

(3) Ocular Defects.-Classically the lenses are dislocated, usually symmetrically upwards and outwards, though other forms do occur. In addition, spherophakia and lenticular myopia may occur, with axial myopia and myopic degeneration at the posterior pole of the globe occurring less commonly. Hypermetropia has been recorded, but seems to be infrequent. Associated with the ectopia of the lenses, is iridodonesis, which may be partial or extend over the whole of the iris. The pupils are usually small and are said to dilate poorly with mydriatics, because of the poor development of the dilator pupillae muscle. The dislocation is usually incomplete, but may develop into a complete dislocation with resultant glaucoma if the lens comes forward into the anterior chamber. Lens opacities and notchings of the lens margin are often found, and elongation of the zonular fibres or deficiency of the zonule may be present. Strabismus (either convergent or divergent) has been reported in Marfan's syndrome, as also quite a high incidence of corectopia and persistent pupillary membrane. Colobomata of the iris, choroid, and disc have also been reported, though these malformations are rare.

Presence of Arachnodactyly.-The difficulty in the cases presented here is that the patients do not seem to show arachnodactyly. We do know however that Marfan's syndrome can be present without the skeletal defects, or without the ocular or cardio-vascular defects - the so-called "forme fruste" types (Reynolds, 1950). In fact, it has been suggested that aortic aneurysms for which no other cause could be found should be regarded as a type of Marfan's syndrome, and that the relatives of cases should be examined to exclude the condition. The determining factor producing these types has been suggested to be a disturbance in the time of the orderly sequence of developmental events during the first few months of intrauterine life (Lambie, Shellshear, and Shellshear, 1950). One may also consider these forms as the result of a single dominant autosomal factor, the effects of which are sensitive to modification by other inherited genetic modifiers and perhaps to factors in the early environment (Lutman and Neel, 1949). If we do accept these cases as presenting a variety of Marfan's syndrome, we are confronted with the problem of the age of onset of the symptoms. The ocular signs and symptoms of Marfan's syndrome are usually well established at a much younger age than they occur in this family, and do not seem to have a progressive nature such as those described here. Ectopia lentis may be traumatic in origin, but that aetiological factor would hardly account for the dislocated lenses in this family unless one postulates minor traumata on a previously weakened zonular structure, i.e., a congenital background. Ectopia lentis may be secondary to some ocular disease 
or degeneration-hardly a likely cause in several members of a family. So we are left with an aetiology based on development.

Clarke (1939) subdivides congenital dislocation of the lens as follows:

(1) Simple.-There is a defective zonule and ciliary body, and apart from the dislocation of the lens, the eye is grossly normal.

(2) Associated with anomalies of ocular dimension, e.g., ectopia with axial myopia, microphthalmos, or buphthalmos.

(3) Associated with anomalies of ocular structure, e.g., persistent pupillary membrane, corectopia, aniridia, polycoria, coloboma of iris, choroid, or lens, and megalocornea.

(4) Associated with congenital anomalies elsewhere in the body, e.g., dwarfism or arachnodactyly.

The family here described would seem to belong to Clarke's Group 1, and the findings of glaucoma, cataract, and detachment are then considered to be the complications and not the cause of the ectopia. In this classification, however, the condition is referred to as congenital with defects in the suspensory mechanism of the lens. In other series of cases described, the ocular defects first became manifest at a much younger age than in this family. Ectopia has been reported in cases of Marfan's syndrome at the age of 18 months, and is often referred to as being first noticed when the child goes to school. In this family going to school does not seem to have brought to light any ocular weaknesses.

Lloyd (1948) subdivides cases of ectopia lentis into two groups:

(1) Non-progressive cases,

(2) Cases that later develop poor vision, progressive displacement of the lenses, choroidal atrophy, and vitreous opacities.

This second group he classes as an abiotrophy which can already be recognized at the age of 10 years. Possibly then, in our cases the lesions could be classed as abiotrophies rather than as congenital defects. The possibility of the postnatal development of true arachnodactyly in a previously normal person has not been solved, but the probability of such an occurrence is extremely remote. We know of other abiotrophies occurring in the eye, and I see no reason why this should not be responsible for the development of ectopia lentis in what was previously apparently a normal eye.

Falls and Cotterman (1943) examined a family of some 119 members, of whom 25 had ectopia lentis, and none showed sufficient deformities to permit of a diagnosis of arachnodactyly. The condition was inherited as a dominant gene, so that about 50 per cent. of the offspring of an affected parent were affected. Some of the cases showed parents who had no obvious abnormal ocular signs, and it was postulated that in these normal parents there had been a lack of penetrance of the gene responsible for the ectopia lentis. This family showed no corectopia and the pupils dilated well with mydriatics. Glaucoma was a frequent occurrence, resulting from either luxation of the lens into the anterior chamber, or of a type more akin to the usual primary non-congestive type. They also found that the dislocated lenses were predisposed to cataract formation. They recommend early refraction to avoid the occurrence of amblyopia and squint, and routine miotics to counteract the tendency for dislocation of the lens into the anterior chamber. 
Needling of these lenses gave poor results, and in the cases of non-acute glaucoma cyclodialysis seemed to be the best of the decompression operations.

Harshman (1948) reports a family to which the conditions found in our cases approximate closely. Three generations were affected and, of the ten members who had glaucoma, six had dislocated lenses and a seventh an incipient cataract. These patients first noted the onset of symptoms during the fourth to sixth decade of life, and these began with the development of myopia or a relatively sudden increase in the degree of myopia. After these refractive changes, glaucoma developed with an acute onset. This type of glaucoma differs greatly from the usual hereditary glaucoma of the non-congestive, insidious, and chronic type. Harshman achieved the best results by performing a basal iridectomy followed by extraction of the lens.

Clarke (1939), however, points out the danger of vitreous loss, which occurred in five out of ten cases subjected to preliminary iridectomy, and in seven of ten extractions done after a preliminary iridectomy. Seven of his ten cases showed visual improvement after operation. Clarke advised that 2 months should elapse between operations, and recommended that the cataract be removed by a scoop extraction. The ultimate prognosis of the untreated cases was poor.

Therapy.-There seems to be little doubt that the glaucoma in the family reported above results from subluxated lenses, and the answer would appear to be to extract the lenses, once symptoms have become apparent. Watching the progress of III, 10, one wonders what signs and symptoms should make one advise operation. Until November, 1952, I had thought that one could wait until the symptoms of glaucoma made themselves evident, and I expected the anterior chambers to become still deeper. Now I wonder whether extremely shallow anterior chambers do not evolve before the glaucoma symptoms supervene, and whether an emergency operation might not then give such good results. I doubt whether I should be bold enough to extract the lens in such an emergency unless it were completely in the anterior chamber. One is loth to extract a clear but subluxated lens, through which a corrected visual acuity of $6 / 6$ or $6 / 9$ can still be obtained, on the chance of averting glaucomatous attacks. I had anticipated that the nutrition of the lens might be impaired by its altered position and physical conditions, so that cataractous changes were produced, before glaucoma supervened. Then, as vision deteriorated as the result of cataract, one would feel better justified in extracting the lens, achieving thereby the dual result of removing the opacifying lens and also the potential cause of glaucoma.

There is no reason why conservative treatment of a glaucomatous attack should not be successful; it has been so in the case of IV, 12, though we must remember that the lens may become impacted in the pupil, producing a "glaucoma inversum". If conservative treatment is satisfactory, should one later advocate surgical intervention? Probably it would be better to do so. The operation of choice appears to be a cyclodialysis, as a trephine may so easily become blocked by the vitreous which is often present in the anterior chamber in cases of dislocated lens, and was in fact noted in III, 10. The cyclodialysis is not a very traumatizing operation; it can be repeated if 
need be in another quadrant of the eye, and is of no embarrassment if extraction of the lens later becomes necessary. On the whole therefore, one should perhaps assume that a decompression operation should be undertaken prophylactically when the anterior chamber shows definite signs of becoming shallower, unless glaucoma symptoms have previously occurred. Then, as the lens becomes opaque, extraction will have to be undertaken. The technique employed for this depends on individual preference, the strong possibility of vitreous being present in the anterior chamber, and the tendency for loss of vitreous in extracting a dislocated lens being higher than with an ordinary cataractous lens being borne in mind.

What of the other members of the family in whom no suspicious signs have yet been seen? I do not think one can give any assurance that the condition will not occur as they get older. It is hard to say at what age the first signs most often appear-the fourth and fifth decades of life seem to be most common, although some cases have started earlier. The only advice one can give is to have their eyes examined at intervals, so that if any sudden increase in myopia should occur they may be seen at 2- or 3-monthly intervals until the diagnosis of subluxated lens can be confirmed or rejected.

\section{Summary}

A family is described in which ectopia lentis with complications affecting twelve persons has been traced through four generations. Various other members of the family have doubtfully healthy eyes and more cases may develop as the years pass. The development of the condition is followed in one patient, and the supervision of such cases is discussed.

I should like to acknowledge the help I have received in the preparation of this paper from my father, Dr. R. C. J. Meyer, who first drew my attention to this interesting family. I am also grateful to Dr. H. J. Hamelberg, who allowed me to see IV, 12, one of the cases described above.

\section{REFERENCES}

Clarke, C. C. (1939). Arch. Ophthal., (Chicago), 21, 124.

Falls, H. F., and Cotterman, C. W. (1943). Ibid., 30, 610.

FISCHL, A. A., and RUTHBERG, J. (1951). J. Amer. med. Ass., 146, 704.

GRAEFE, A. L. vON (1854). v. Graefes Arch. Ophthal., 1, pt. 1, 336.

HAMWI, G. J. (1951). Amer. J. Med., 11, 261.

HARShMAN, J. P. (1948). Amer. J. Ophthal., 31, 833.

HeAtH, P. (1941). Arch. Ophthal. (Chicago), 25, 424.

Lambie, C. G., Shellshear, K. E., and ShellShear, J. L. (1950). Med. J. Austr., 1, 213.

Lindeboom, G. A., and Westerveld-Brandon, E. R. (1950). Cardiologia (Basel), $17,217$.

LLOYD, R. I. (1948). Arch. Ophthal. (Chicago), 40, 558.

LUTMAN, F. C., and NeEL, J. V. (1949). Ibid., 41, 276.

MARVEL, R. J., and GeNOVESE, P. D. (1951). Amer. Heart J., 42, 814.

MORTON, A. S. (1879). Roy. Lond. ophthal. Hosp. Rep., 9, 435.

RADOS, A. (1942). Arch. Ophthal. (Chicago), 27, 477.

REYNolds, G. (1950). Guy's Hosp. Rep., 99, 178.

Ross, L. J. (1949). Amer. J. Dis. Child, 78, 417. 